\section{OPEN ACCESS}

Edited by:

Youhui Lin,

Xiamen University, China

Reviewed by: Jose Eltit,

Virginia Commonwealth University,

United States

Peter Hamilton,

Virginia Commonwealth University,

United States

*Correspondence:

Elena Bossi

elena.bossi@uninsubria.it Ivet Bahar

bahar@pitt.edu

${ }^{+}$These authors have contributed equally to this work

Specialty section:

This article was submitted to

Chemical Biology,

a section of the journal

Frontiers in Chemistry

Received: 05 August 2021

Accepted: 16 November 2021

Published: 10 December 2021

Citation:

Romanazzi T, Zanella $D$, Cheng $M H$, Smith B, Carter AM, Galli A, Bahar I and Bossi E (2021) Bile Acids Gate

Dopamine Transporter Mediated Currents.

Front. Chem. 9:753990.

doi: $10.3389 /$ fchem.2021.753990

\title{
Bile Acids Gate Dopamine Transporter Mediated Currents
}

\author{
Tiziana Romanazzi ${ }^{1+}$, Daniele Zanella ${ }^{2 \dagger}$, Mary Hongying Cheng ${ }^{3+}$, Behrgen Smith ${ }^{4}$, \\ Angela M. Carter ${ }^{2}$, Aurelio Galli ${ }^{2}$, Ivet Bahar ${ }^{3 *}$ and Elena Bossi ${ }^{1,5 *}$
}

${ }^{1}$ Department of Biotechnology and Life Sciences, University of Insubria, Varese, Italy, ${ }^{2}$ Department of Surgery, University of Alabama at Birmingham, Birmingham, AL, United States, ${ }^{3}$ Department of Computational and Systems Biology, School of Medicine, University of Pittsburgh, Pittsburgh, PA, United States, ${ }^{4}$ Department of Physics and Chemistry, Biomolecular Engineering, Milwaukee School of Engineering, Milwaukee, WI, United States, ${ }^{5}$ Center for Research in Neuroscience, University of Insubria, Varese, Italy

Bile acids (BAs) are molecules derived from cholesterol that are involved in dietary fat absorption. New evidence supports an additional role for BAs as regulators of brain function. Sterols such as cholesterol interact with monoamine transporters, including the dopamine (DA) transporter (DAT) which plays a key role in DA neurotransmission and reward. This study explores the interactions of the BA, obeticholic acid (OCA), with DAT and characterizes the regulation of DAT activity via both electrophysiology and molecular modeling. We expressed murine DAT (mDAT) in Xenopus laevis oocytes and confirmed its functionality. Next, we showed that OCA promotes a DAT-mediated inward current that is $\mathrm{Na}^{+}$-dependent and not regulated by intracellular calcium. The current induced by OCA was transient in nature, returning to baseline in the continued presence of the BA. OCA also transiently blocked the DAT-mediated $\mathrm{Li}^{+}$-leak current, a feature that parallels DA action and indicates direct binding to the transporter in the absence of $\mathrm{Na}^{+}$. Interestingly, OCA did not alter DA affinity nor the ability of DA to promote a DAT-mediated inward current, suggesting that the interaction of OCA with the transporter is non-competitive, regarding DA. Docking simulations performed for investigating the molecular mechanism of OCA action on DAT activity revealed two potential binding sites. First, in the absence of DA, OCA binds DAT through interactions with D421, a residue normally involved in coordinating the binding of the $\mathrm{Na}^{+}$ion to the $\mathrm{Na} 2$ binding site (Borre et al., J. Biol. Chem., 2014, 289, 25764-25773; Cheng and Bahar, Structure, 2015, 23, 2171-2181). Furthermore, we uncover a separate binding site for OCA on DAT, of equal potential functional impact, that is coordinated by the DAT residues R445 and D436. Binding to that site may stabilize the inward-facing (IF) open state by preventing the re-formation of the IFgating salt bridges, R60-D436 and R445-E428, that are required for DA transport. This study suggests that BAs may represent novel pharmacological tools to regulate DAT function, and possibly, associated behaviors.

Keywords: bile acid, dopamine, monoamines, transporters, SLC6, electrophysiology 


\section{INTRODUCTION}

Bile acids (BAs) are amphipathic molecules derived from cholesterol that are primarily synthesized in the liver and stored in the gallbladder. Upon food consumption and transit, BAs are released into the duodenum (Mertens et al., 2017) where their main physiological role is solubilization and absorption of dietary fat. Administration of BAs has been developed into successful therapies for the treatment of liver and gallbladder pathologies, such as nonalcoholic steatohepatitis and cholelithiasis (Cruz-Ramon et al., 2017; Li and Chiang, 2020). When administered orally, they exhibit favorable bioavailability. They are readily absorbed through the portal vasculature and distributed throughout the body (Kiriyama and Nochi, 2019; Fiorucci et al., 2021).

Receptors for BAs are present throughout the brain (Maruyama et al., 2002; Kawamata et al., 2003; Keitel et al., 2010; Huang et al., 2015; Perino et al., 2021) and evidence exists for their synthesis directly in the CNS (McMillin and DeMorrow, 2016; Mertens et al., 2017; Kiriyama and Nochi, 2019). This raises the possibility of physiologic roles for BAs other than acting as an adjuvant in fat absorption, such as regulators of CNS activity. Consistent with this idea, freely circulating BAs have been implicated in the modulation of CNS proteins such as NMDA, $\mathrm{GABA}_{\mathrm{A}}$ and $\mathrm{M}_{3}$ muscarinic receptors (Raufman et al., 2002; Schubring et al., 2012), the activation of neuronal ion channels (Wiemuth et al., 2014; Kiriyama and Nochi, 2019) and stimulation of the release of neuroactive peptides such as GLP-1 (Flynn et al., 2019; Chaudhari et al., 2021; Fiorucci et al., 2021). BAs pass the blood-brain barrier (BBB) through passive diffusion, as well as through active membrane transporters, and BA levels in the brain have been correlated to plasma levels (Mano et al., 2004; Higashi et al., 2017; Reddy et al., 2018; Kiriyama and Nochi, 2019). The number of studies proposing BAs as a treatment for brain disorders are steadily increasing (Bhargava et al., 2020; Wu et al., 2020; Huang et al., 2021; Jin et al., 2021; Zangerolamo et al., 2021) but understanding of their mechanisms of action is still limited.

BA concentrations vary in the body following a circadian rhythm and are highly influenced by the fasting vs fed state (Fiorucci et al., 2021; Perino et al., 2021). Changes in circulating BAs are also promoted by pathological alterations in liver functionality and metabolism (Fiorucci et al., 2021). Multiple types of bariatric surgery increase circulating BAs and, in mice, cause loss of preference for dietary fat (Scholtz et al., 2014; Bensalem et al., 2020) and, interestingly, cocaine (Reddy et al., 2018). Further, feeding BAs to mice recapitulated the effects of bariatric surgery on cocaine preference, strongly supporting their ability to modulate reward circuitries in the CNS as well as dopamine (DA) neurotransmission (Reddy et al., 2018). Understanding the molecular determinants of these actions could facilitate BA-based therapies for drug use disorders as well as identify potential new therapeutic targets.

BAs are directly derived from cholesterol and retain a high degree of structural similarity with the parent molecule but differ by the presence of various $\mathrm{R}$ groups, many of which are charged, added to the $\mathrm{A}, \mathrm{B}$, and $\mathrm{C}$ rings as well as the hydrocarbon tail. Resulting BAs are less lipophilic and more soluble in aqueous solutions relative to cholesterol (Russell, 2003). The importance of cholesterol in monoamine transporter (MAT) function and membrane localization is well documented (Foster et al., 2008; Chang and Rosenthal, 2012). Cholesterol depletion modulates both MAT expression (Butchbach et al., 2004; Magnani et al., 2004; Foster et al., 2008) and activity (Magnani et al., 2004; Adkins et al., 2007; Foster et al., 2008; Hong and Amara, 2010). There are indications of direct interactions between cholesterol and MATs such as the DA transporter (DAT) and human serotonin transporter (hSERT) (Cremona et al., 2011; Jones et al., 2012; Penmatsa et al., 2013; Wang et al., 2015; Coleman et al., 2016; Zeppelin et al., 2018) whereas no information is available on interactions occurring between BAs and MATs. Thus, investigations of the functional relevance of interactions between cholesterol-like molecules and MATs are needed to further define the central regulatory role of these sterols. This study demonstrates the ability of BAs to alter DAT activity and identifies putative binding sites that may underlie the structural changes driving these alterations.

\section{MATERIALS AND METHODS}

\section{Solutions}

Composition of buffered solution (ND96) (in mM): $\mathrm{NaCl} 96, \mathrm{KCl}$ 2, $\mathrm{CaCl}_{2}$ 1.8, $\mathrm{MgCl}_{2}$ 1, HEPES 5, pH 7.6. Composition of NDE solution: ND96 plus $2.5 \mathrm{mM}$ pyruvate and $50 \mu \mathrm{g} / \mathrm{ml}$ Gentamycin sulphate. Composition of external control buffer (ND98) (in $\mathrm{mM}$ ): $\mathrm{NaCl} 98, \mathrm{MgCl}_{2} 1$, and $\mathrm{CaCl}_{2} 1.8$ with or without $0.01 \%$ DMSO. In tetramethylammonium (TMA)-chloride "zero sodium” buffer (TMA98), equimolar TMACl replaces $\mathrm{NaCl}$. In $\mathrm{Li}^{+}$buffer, equimolar $\mathrm{LiCl}$ replaces $\mathrm{NaCl}$. The final $\mathrm{pH}$ was adjusted using respective hydroxides $(\mathrm{NaOH}$ or TMAOH or $\mathrm{LiOH})$ to 7.6 for all external solutions. Substrates used were Dopamine (DA) (Calbiochem - Sigma, Milan, Italy), Lithocholic acid (LCA) (Sigma), and Obeticholic acid (OCA) (Adipogen, Switzerland). LCA or OCA powder was dissolved in DMSO at $50 \mathrm{mM}$ and $100 \mathrm{mM}$, respectively.

\section{Oocytes Collection and Preparation}

Oocytes were obtained from adult Xenopus laevis females. Animals were anaesthetised in $0.1 \%(\mathrm{w} / \mathrm{v}) \mathrm{MS} 222$ (tricaine methanesulfonate; Sigma) solution in tap water. Abdomens were sterilized with antiseptic agent (Povidone-iodine 0.8\%), laparotomy was performed, and portions of the ovary were collected. The oocytes were treated with $0.5 \mathrm{mg} / \mathrm{ml}$ collagenase (Sigma Type IA) in ND96 calcium-free for at least $30 \mathrm{~min}$ at $18^{\circ} \mathrm{C}$. Healthy and fully-grown oocytes were selected and stored at $18^{\circ} \mathrm{C}$ in NDE solution (Bossi et al., 2007). The day after the removal, the oocytes were injected with cRNA using a manual microinjection system (Drummond Scientific Company, Broomall, PA). Injected concentrations were $12.5 \mathrm{ng} / 50 \mathrm{nl}$ for the mouse dopamine transporter (mDAT), $2 \mathrm{ng} / 50 \mathrm{nl}$ for human Takeda $\mathrm{G}$ proteincoupled receptor (hTGR5).

\section{cRNA Preparation}

mDAT cDNA in pcDNA3.1 was kindly gifted from Prof. Dr. Harald Sitte of Medical University of Vienna. The cDNA mDAT 
was amplified with forward and reverse primers containing SmaI and EcoRI restriction sites, respectively (5'-GACTCCCGGGACC CATGAGTAAAAGCAAATG-3'; $5^{\prime}$-GCATGAATTCTTACAG CAACAGCCAATGGCGC- $3^{\prime}$ ). The amplified coding sequence was then subcloned into the $\mathrm{pGHJ}$ vector after double digestion with SmaI e EcoRI restriction enzymes (Promega). hTGR5 gene was in pCMV6-Entry (GPBAR1 Human cDNA ORF Clone, NM_001077191; Origene Technologies, Inc., Rockville, MD, United States). The two plasmids were linearized with SalI (mDAT) and with NdeI (hTGR5), in vitro capped, and transcribed using T7 RNA polymerase. Enzymes were supplied by Promega Italia. The oocytes were incubated at $18^{\circ} \mathrm{C}$ for 2-3 days prior to electrophysiological experiments. The experimental protocol was approved locally by the Committee of the "Organismo Preposto al Benessere degli Animali" of the University of Insubria (OPBA-permit \#02_15) and nationally by Ministero della Salute (permit nr. 1011/2015).

\section{Electrophysiology}

Electrophysiological studies were performed using the TwoElectrode Voltage Clamp (TEVC) technique (Oocyte Clamp OC725; Warner Instruments, Hamden, CT, United States). Controlling software was WinWCP version 4.4.6 (J. Dempster, University of Strathclyde, United Kingdom) or Clampex (Molecular Devices, Sunnyvale, CA, United States, www.moleculardevices.com). Borosilicate microelectrodes, with a tip resistance of $0.5-4 \mathrm{M} \Omega$, were filled with $3 \mathrm{M} \mathrm{KCl}$. Bath electrodes were connected to the experimental oocyte chamber via agar bridges ( $3 \%$ agar in $3 \mathrm{M} \mathrm{KCl})$. The holding potential was kept at $-60 \mathrm{mV}$ for all the experiments. The minimal concentration of OCA inducing inward current was evaluated by applying concentration of OCA from $1 \mathrm{pM}$ to $100 \mu \mathrm{M}$. Each dose was tested on one single oocyte, exposed before to $30 \mu \mathrm{M}$ DA. The current in the presence of OCA is the maximal current measured at the peak. For the I/V relationships, a step protocol from $-160 \mathrm{mV}$ to $+40 \mathrm{mV}$ with $20 \mathrm{mV}$ of increment was applied for $100 \mathrm{~ms}$. The mean of the transport-associated currents plotted in the scatter diagrams and in the I/V relationships were determined by subtracting the current recorded in the ND98 buffer from the current recorded in the presence of DA or OCA. In experiments using TMA, subtraction was performed with the current in TMA98 buffer. To chelate intracellular calcium, oocytes were injected with $50 \mathrm{nl}$ of an intracellular solution containing $13 \mathrm{mM}$ EGTA $30 \mathrm{~min}$ prior to electrophysiological recording. Intracellular solution had the following composition (in $\mathrm{mM}$ ): $\mathrm{KCl} 130, \mathrm{NaCl} 4, \mathrm{MgCl}_{2}$ 1.6, HEPES 10, glucose 5, pH 7.6. The dose-response experiments in Figure 4 were performed exposing the same oocytes first to all the concentrations of DA alone, then to the same concentrations plus $10 \mu \mathrm{M}$ OCA. Data analysis was performed using Clampfit 10.2 software (Molecular Devices, Sunnyvale, CA, United States, www.moleculardevices.com); OriginPro 8.0 (OriginLab Corp., Northampton, MA, United States, www.originlab.com) and GraphPad Prism (www.graphpad.com/scientific-software/prism) were used for statistical analysis and figure preparation.

\section{Structural Models for mDAT and hDAT}

The mDAT (residues R56 to N596; UniProt ID O55192) in the outward-facing open ( $\mathrm{OFo}$ ), occluded, and inward-facing open (IFo) states were generated using SWISS-MODEL (Waterhouse et al., 2018) based on the structures resolved for OFo Drosophila DAT (dDAT) (PDB: 4M48) (Penmatsa et al., 2013), occluded hSERT (PDB: 6DZV) and IFo hSERT (PDB: 6DZZ) (Coleman et al., 2019). Homology models for hDAT in these conformational states were taken from previous work (Cheng et al., 2018; Aggarwal et al., 2021).

\section{Docking Simulations}

OCA 3D structures were downloaded from the ZINC database (Irwin and Shoichet, 2005) (ZINC14164617) and DrugBank (Wishart et al., 2006) (DB05990). The net charge of OCA is indicated to be -1 in the ZINC database, and zero in DrugBank. Docking simulations were performed using both electronic states, designated as $\mathrm{OCA}(-)$ and $\mathrm{OCA}(\mathrm{n})$ for the negatively-charged and neutral OCAs, respectively. The binding sites and binding poses on both mDAT and hDAT, in different conformational states, were assessed using docking simulation software AutoDock4 (Morris et al., 2009) and AutoDock Vina (Trott and Olson, 2010). Autodock Vina simulations were carried out using a grid with dimensions set to $84 \times 58 \times 86 \AA^{3}$ and center at $(-1.22,1.16,-6.26 \AA)$ for each conformer and each transporter. This grid box encapsulated the entire structure of the transporter. The number of runs (exhaustiveness parameter in Autodock Vina) was set to 50 and the algorithm returned 20 binding modes of interest for each conformer. AutoDock4 simulations were performed following previously published protocols (Cheng et al., 2015; Aggarwal et al., 2021). Briefly, Lamarckian genetic algorithm with default parameters was employed with the maximal number of energy evaluations set to $2.5 \times 10^{7}$. The binding energy was estimated from the weighted average of multiple binding poses at a given site observed in 100 independent runs.

\section{RESULTS}

\section{OCA Induces a DAT-Dependent, Transient Inward Current}

To begin to investigate the regulatory effects of OCA on DAT electrical activity, Xenopus laevis oocytes were tested by twoelectrode voltage clamp (TEVC) (Figure 1A). Perfusion of DA onto the oocytes expressing the mouse DAT (mDAT) generated an inward current $(-12.5 \mathrm{nA} \pm 0.63)$ (Figures 1B,C, left), confirming the expression and functionality of the transporter. On the same oocytes, following DA washout, perfusion of $10 \mu \mathrm{M}$ OCA also generated an inward transport current $(-8.28 \mathrm{nA} \pm$ 0.6 ). This membrane conductance exhibited a lower amplitude and inactivated rapidly (Figures 1B,C, left). The OCA-induced transient inward current was also elicited when OCA was perfused prior to DA exposure (data not shown).

Next, we set out to understand whether the OCA current was mediated by endogenous targets of OCA. One of the main targets of OCA, the G-Protein Coupled Receptor TGR5, is shown to be only minimally expressed in Xenopus laevis oocytes as reported in the platform Xenbase (Yanai et al., 2011; Session et al., 2016; JamesZorn et al., 2018). As shown in Figure 1B, no response from the perfusion of OCA was obtained in control oocytes (i.e., not expressing any heterologous protein). Thus, an activation of 


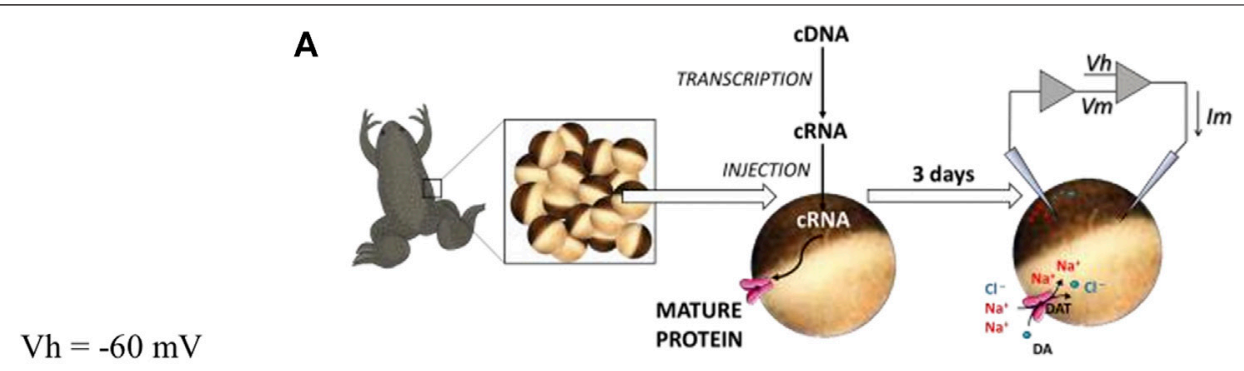

B

mDAT

mDAT-hTGR5

UNINJECTED

hTGR5
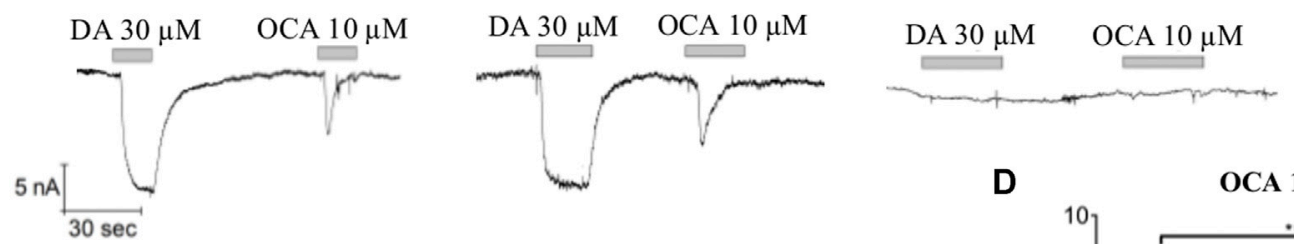

OCA $10 \mu \mathrm{M}$
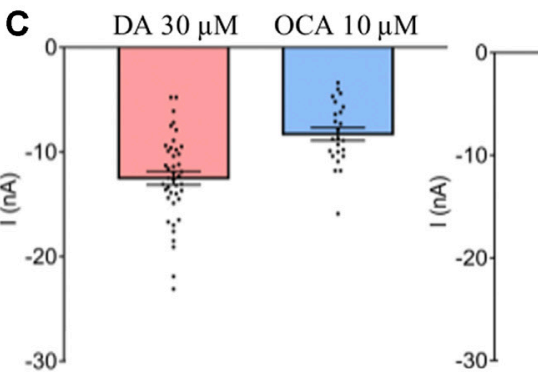

DA $30 \mu \mathrm{M} \quad$ OCA $10 \mu \mathrm{M}$
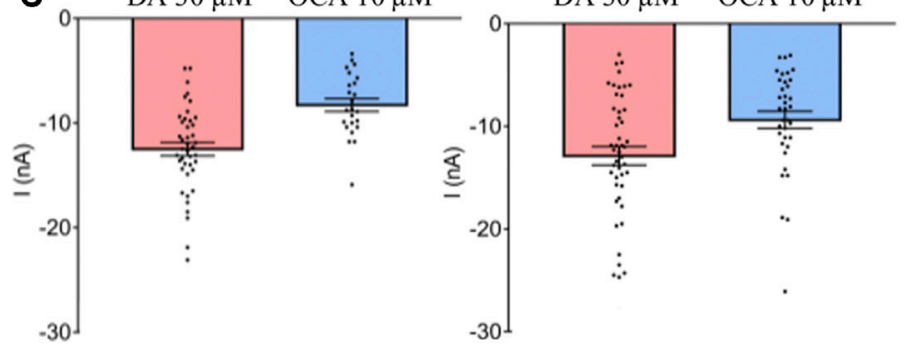

D

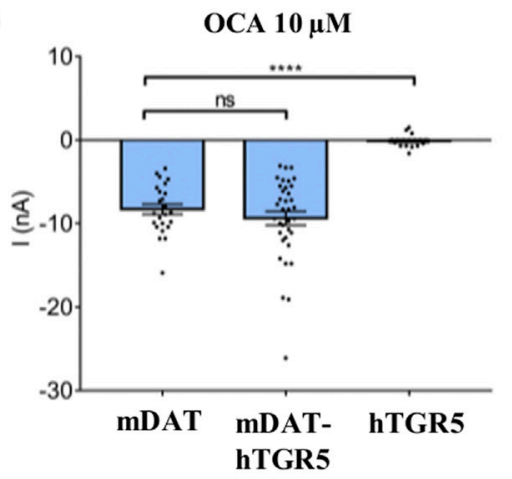

E

$1 \mathrm{pM}$
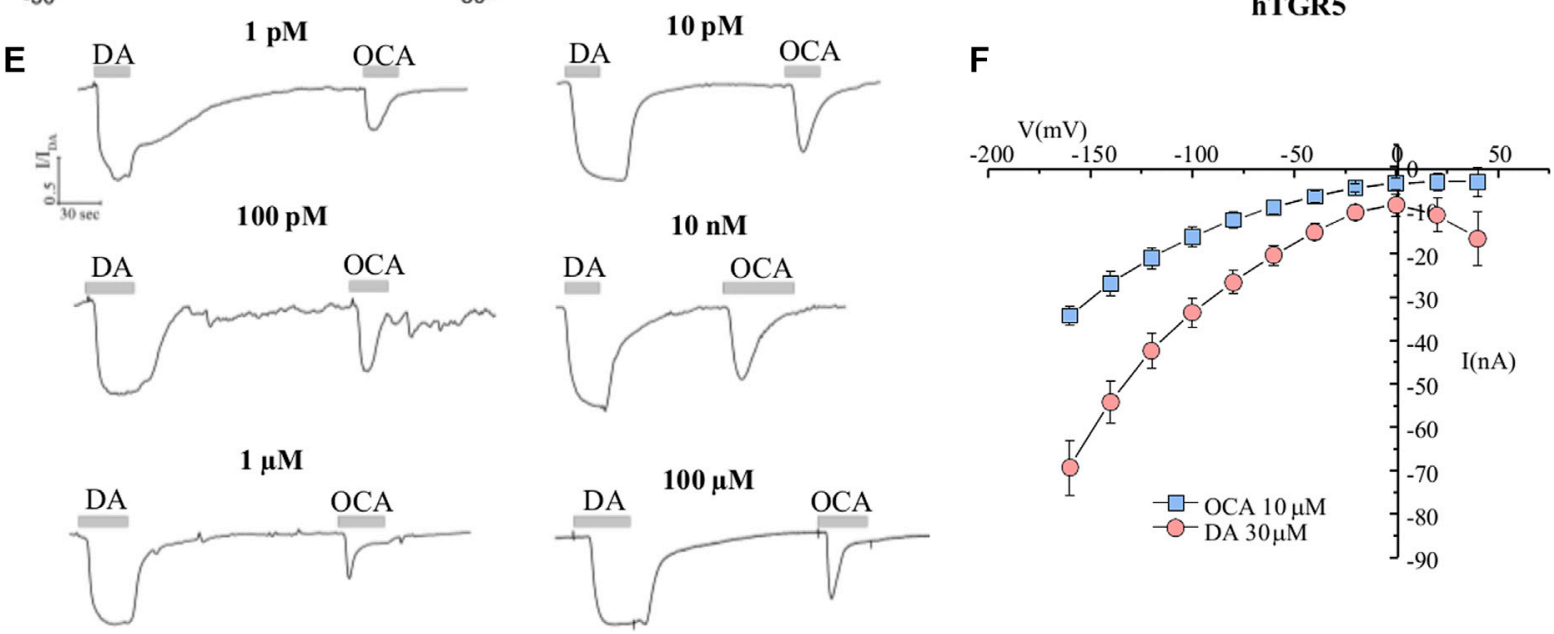

FIGURE 1 | OCA generates an electrical current through the DAT. (A) Schematic representation of oocyte collection, cRNA synthesis and injection, and TEVC technique. (B) Representative traces of currents recorded by TEVC $(\mathrm{Vh}=-60 \mathrm{mV})$ from oocytes expressing mDAT, without or with hTGR5, uninjected, or expressing hTGR5 alone. The oocytes were perfused with $30 \mu \mathrm{M}$ DA or $10 \mu \mathrm{M}$ OCA. (C) Mean of the maximal DA-associated and OCA-induced currents (I nA \pm SE of $14-47$ oocytes, 6-11 batches) in oocytes expressing mDAT (C, left) or mDAT plus TGR5 (C, right). (D) Mean of the maximal OCA-induced currents in oocytes expressing the proteins indicated; one-way ANOVA F $(2,74)=36.13, p<0.0001$ followed by Tukey's multiple comparison test (mDAT vs mDAT-hTGR5 $p=0.566, \mathrm{mDAT}$ vs hTGR5 $p<0.0001)$. (E) Representative traces of currents recorded by TEVC $(\mathrm{Vh}=-60 \mathrm{mV})$ from oocytes tested with DA $(30 \mu \mathrm{M})$ and OCA at the indicated concentrations. The current of each trace was normalized to the mean current of dopamine to reduce the variability between batches. (F) IN relationships of DA (30 $\mu M$ ) and OCA $(10 \mu \mathrm{M})$-induced currents.

endogenous targets seems unlikely. As previously reported in literature, oocytes can express functional human TGR5 (hTGR5) (Lieu et al., 2014) as demonstrated by modulation of various membrane proteins upon TGR5 activation. We expressed
hTGR5 in oocytes and measured OCA-induced currents. No currents were observed in hTGR5 expressing oocytes upon OCA perfusion (Figure 1B, right and Figure 1D). Next, we tested oocytes co-expressing mDAT and hTGR5. The currents 
recorded from these cells in response to $\mathrm{DA}(-12.88 \mathrm{nA} \pm 0.9)$ and OCA ( $-9.37 \mathrm{nA} \pm 0.82$ ) alone (Figure 1B, center and Figure 1C, right) were comparable to the ones recorded in oocytes expressing mDAT alone (Figure 1D). This data suggests that the inward current induced by OCA is directly mediated by mDAT.

Dose-response experiments determined that $10 \mu \mathrm{M}$ OCA was a saturating concentration. The EC50 was not calculated for this interaction because the current amplitude was consistent in response to over an eight order of magnitude range of OCA concentrations, as low as $1 \mathrm{pM}$ and as high as $100 \mu \mathrm{M}$ OCA (Figure 1E). The concentration of $10 \mu \mathrm{M}$ was chosen throughout this study because it approximates the concentration of OCA in the plasma after oral administration and is sufficient to activate physiological targets of OCA in vitro (Roda et al., 2014).

To better characterize the OCA-induced current, we performed a voltage step protocol and obtained I/V curves for the transporter with DA and OCA (Figure 1F). As expected, DA-induced currents increased at negative potential without reverting. At positive voltages, the I/V curve for DA shows the distinctive inverted $U$ shape, previously reported by Sonders and coworkers (Sonders et al., 1997), which is a hallmark of blockage of the tonic $\mathrm{Na}^{+}$-leak current. OCA displays a similar curve shape. However, it shows an increase in conductance at negative potentials and a lack of inversion at positive potentials (Figure 1F). These characteristics point towards OCA opening a conductance in mDAT.

\section{OCA Modulates the DAT $\mathrm{Li}^{+}$-Leak Current}

Several members of the solute carrier 6 (SLC6) family of transporters exhibit basal leak currents (Lester et al., 1994; Bossi et al., 1999; Andrini et al., 2008) that are augmented in the presence of $\mathrm{Li}^{+}$ions. The $\mathrm{Li}^{+}$-leak current can be utilized to highlight the effect of molecules that interact with transporters and modify their electrical activity in the absence of $\mathrm{Na}^{+}$. DAT shows a significant $\mathrm{Li}^{+}$-leak current that is partially blocked upon DA perfusion (Giros et al., 1992; Sonders et al., 1997). Therefore, we used $\mathrm{Li}^{+}$to investigate whether OCA, similarly to DA, binds mDAT in the absence of $\mathrm{Na}^{+}$. Oocytes were perfused first either with DA or OCA in $\mathrm{Na}^{+}$bathing buffer (Figures 2A,B). Switching to $\mathrm{Li}^{+}$bathing buffer induced a large inward current $(-92.41 \mathrm{nA} \pm 7.45)$ (Figures 2A,B). As expected, the addition of DA to the $\mathrm{Li}^{+}$buffer partially blocked the $\mathrm{Li}^{+}$-leak current (Figures 2A,B). This inhibition occurred in two phases; a rapid transient component (current at the peak: $-25.72 \mathrm{nA} \pm 4.07)$ followed by a steady-state condition $(-38.97 \mathrm{nA} \pm 5.11)$. After $\mathrm{DA}$ removal, the $\mathrm{Li}^{+}$-leak current returned to initial values (Figure $2 \mathbf{A}$ ). Interestingly, perfusion of OCA also partially blocked the $\mathrm{Li}^{+}$-leak current (Figures 2A,B). As with DA, this inhibition displayed two phases; a transient rapid component (current at the peak: $-66.86 \mathrm{nA} \pm 6.13$ ) and a steadystate component $(-82.42 \mathrm{nA} \pm 7.69)$. Inhibition of the $\mathrm{Li}^{+}$-leak current is a strong indication of direct binding of OCA to the transporter in the absence of $\mathrm{Na}^{+}$.

\section{mDAT-Mediated OCA Current Is $\mathrm{Na}^{+}$ Dependent}

In addition to a coupled mechanism, $\mathrm{Na}^{+}$also permeates through DAT in the absence of DA. This generates a leak current that can be uncovered when $\mathrm{Na}^{+}$is substituted by non-permeant cations such as choline or $\mathrm{TMA}^{+}$(Sonders et al., 1997). To better understand the effect of OCA on membrane conductance and the relevance of $\mathrm{Na}^{+}$in the OCA-induced inward current, experiments were repeated with $\mathrm{TMA}^{+}$as the cation substituting for $\mathrm{Na}^{+}$in the bathing buffer. TMA ${ }^{+}$blocked the $\mathrm{Na}^{+}$-leak current $(15.12 \mathrm{nA} \pm 1.71$ ) (Figures 2C,D). As expected, in the presence of $\mathrm{TMA}^{+}$, DA elicited only a fast-transient inward current $(-1.98 \mathrm{nA} \pm 0.37)$, confirming that $\mathrm{Na}^{+}$is necessary for mDAT-mediated DA currents. OCA behaved similarly to DA: the inward current was still present but greatly reduced in amplitude $(-1.83 \mathrm{nA} \pm 0.38$ ) (Figures 2C,D). The substitution of $\mathrm{TMA}^{+}$for $\mathrm{Na}^{+}$greatly affected the amplitude of the OCAinduced currents, pointing to a major role for the permeation of $\mathrm{Na}^{+}$ions in causing the OCA-induced currents recorded in physiological solution.

\section{OCA-Induced Current Is Not Due to an Increase in Intracellular (IC) Calcium}

OCA has been shown to induce IC $\mathrm{Ca}^{2+}$ fluctuations (Hao et al., 2017). Thus, it is possible that the OCA-induced inward current could be generated by the activation of chloride conductance due to an increase in IC $\mathrm{Ca}^{2+}$ concentrations. To investigate this possibility, experiments were conducted in oocytes expressing mDAT and injected with the $\mathrm{Ca}^{2+}$-chelating agent EGTA (Figure 3A). The presence of EGTA did not alter mDATmediated currents elicited by DA or OCA (Compare Figure 3B with Figures 1B,C). Maximal OCA-induced inward currents were also not altered by the presence or absence of EGTA (Figure 3C). Together, these data strongly suggest that IC $\mathrm{Ca}^{2+}$ does not regulate OCA-induced currents and further suggest that effects of OCA are mediated by direct interaction with mDAT.

\section{OCA Does Not Alter Either DAT-Mediated DA Currents or DA Affinity}

Data thus far indicate that OCA interacts directly with mDAT in the absence of DA. We next investigated whether OCA regulates DAT-mediated DA-induced currents. Currents generated from increasing concentrations of DA were unaltered by the presence of $10 \mu \mathrm{M}$ OCA even at low DA concentration (Figures $4 \mathrm{~A}, \mathbf{B}$ ). Mean of maximal currents were fitted to a Hill equation (Figure 4C) and the results reported in Figure 4D. These data indicated that the presence of OCA does not significantly affect either the affinity or the maximal transport currents.

\section{Lithocholic Acid Induces DAT-Mediated Currents}

To determine whether the OCA-induced mDAT-mediated current is specific to OCA, or is a common phenotype induced by bile acids, we investigated the effect of the natural bile acid, lithocholic acid (LCA). OCA and LCA share the same sterol-based structure with different $\mathrm{R}$ groups at positions 5 and 6 of the B ring (Figure 5A). Specifically, ethyl 

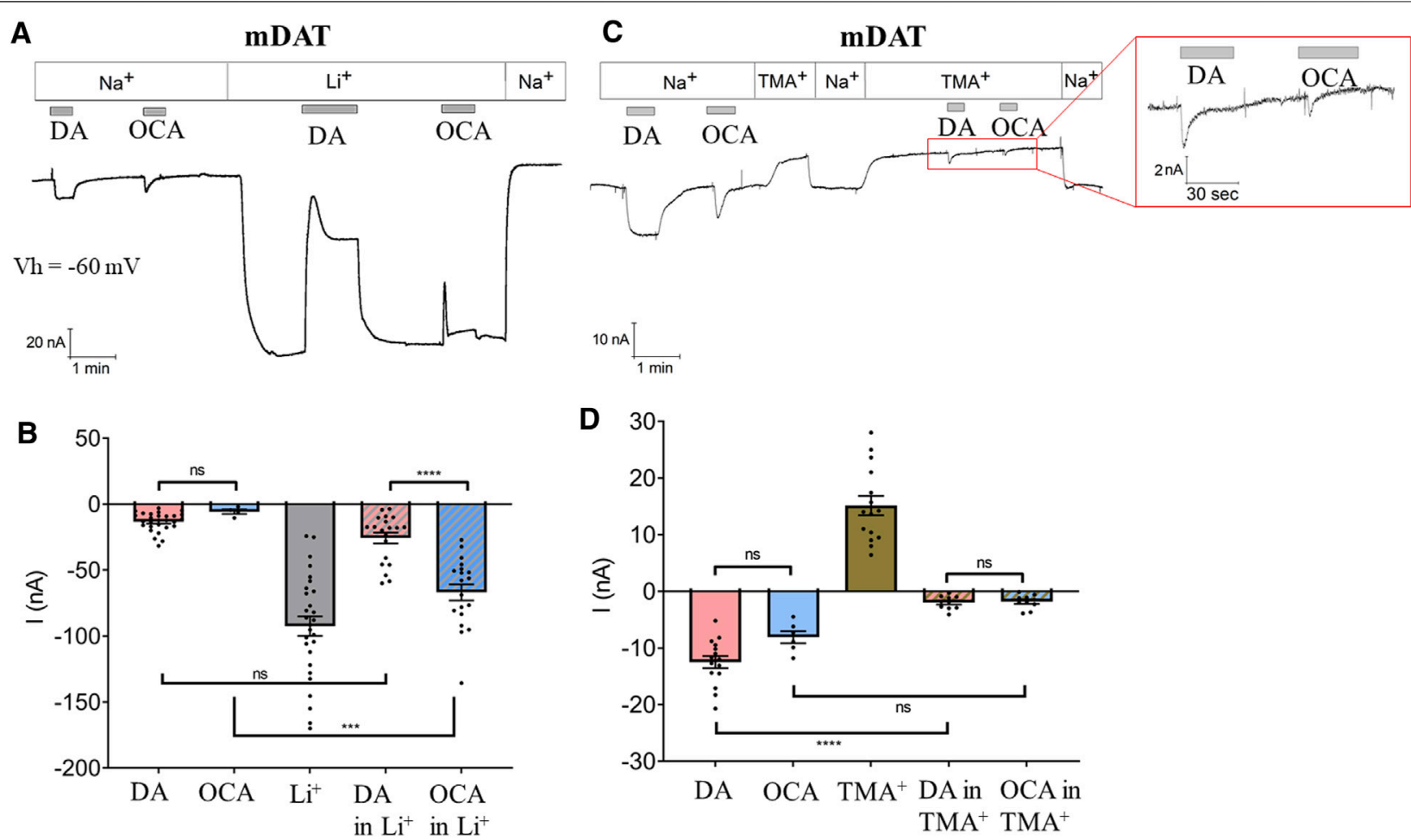

FIGURE 2 | OCA modulates the mDAT-mediated leak current. (A) Representative current traces recorded with TEVC (Vh = -60 mV) in oocytes expressing mDAT perfused with $30 \mu \mathrm{M}$ DA or $10 \mu \mathrm{M}$ OCA in ND98 or Li98 buffer. (B) Mean of maximal currents under conditions shown in A (I nA \pm SE of $6-28$ oocytes, 4 batches. Oneway ANOVA F $(4,94)=42.29, p<0.0001$ followed by Bonferroni's multiple comparison test (DA vs OCA $p>0.999$, DA vs DA in Li ${ }^{+} p>0.999$, OCA vs OCA in Li $p=$ $0.0004, \mathrm{DA}$ in $\mathrm{Li}^{+}$vs OCA in $\left.\mathrm{Li}^{+} p<0.0001\right)$. (C) Representative trace from oocytes expressing mDAT perfused with $30 \mu \mathrm{M}$ DA or $10 \mu \mathrm{M}$ OCA in ND98 or TMA98 buffer. (D) Mean of maximal currents under conditions shown in $\mathrm{C}(\mathrm{nA} \pm \mathrm{SE}$ of $6-15$ oocytes, 3 batches. One-way ANOVA F(4,51) = 86.94, $p<0.0001$ followed by

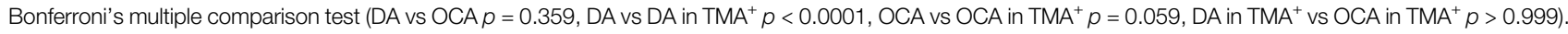

and hydroxyl groups present in OCA are substituted by hydrogen in LCA. Similar to OCA, perfusion of $10 \mu \mathrm{M}$ LCA onto mDAT-expressing oocytes induced a transient inward current $(-10.25 \mathrm{nA} \pm 2.06)$ (Figures 5B,C). Direct comparison of OCA and LCA revealed no significant differences in their ability to induce DAT-mediated currents (Figure 5C). These data suggest that the ability of OCA to promote DAT-mediated currents is shared by another BA.

\section{The Binding Pose of OCA(n) Suggests That It Binds to DAT in a Non-Competitive Fashion, That Allows Simultaneous Binding of DA}

To identify potential sites for binding of OCA onto mDAT and hDAT, docking simulations were performed, using AutoDock4 (Morris et al., 2009) and AutoDock Vina (Trott and Olson, 2010). To account for alternative protonated and deprotonated states of OCA, both the neutral $(\mathrm{OCA}(\mathrm{n}))$ and negativelycharged $(\mathrm{OCA}(-))$ forms (Figure 6D) were used in the simulations. Computations were performed for the outwardfacing open (OFo), occluded (OCC), and inward-facing open (IFo) states of both mDAT and hDAT. The computations revealed that OCA selected similar binding poses, with comparable binding affinities, for either transporter when the same conformational state was targeted regardless of the species. Therefore, representative results for hDAT are presented unless otherwise stated.

Figure 6A illustrates the top-ranking distinct binding poses and sites of both OCA forms, observed in docking simulations, onto hDAT in the OFo and IFo states in the absence of DA. Panel $\mathrm{B}$ shows the comparison to the DA-bound structures resolved for dDAT (Wang et al., 2015) in the presence of OCA, and panel C to ibogaine-bound hSERT (Coleman et al., 2019). Notably, in the case of the OFo conformer, the most favorable binding sites for both the neutral and negatively charged forms of OCA are within the extracellular (EC) vestibule but the exact locations are determined by the protonation state of the ligand. In the absence of DA, OCA(-) occupies the S2 site proposed to allosterically modulate transport (Cheng and Bahar, 2019), whereas OCA(n) binds an extended region spanning between the primary (S1) and secondary (S2) sites (Figure 6A, top panels). The binding pose of OCA(-) thus differs from that of the DA bound to OFo DAT (Figure 6B) where the residue equivalent to hDAT D79 (D46 in dDAT) plays a major role in coordinating the binding of the amine group through a salt bridge formation 


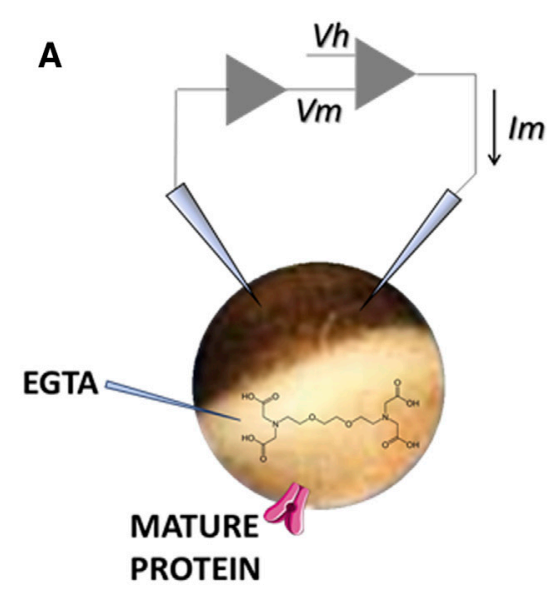

C

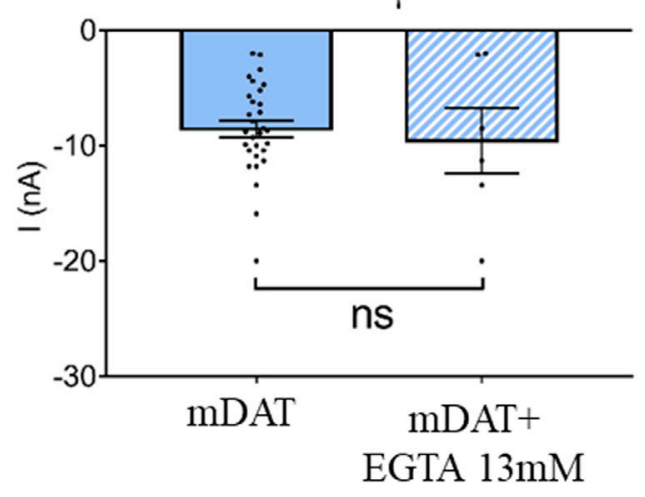

\section{B $\quad$ mDAT + EGTA 13 mM}
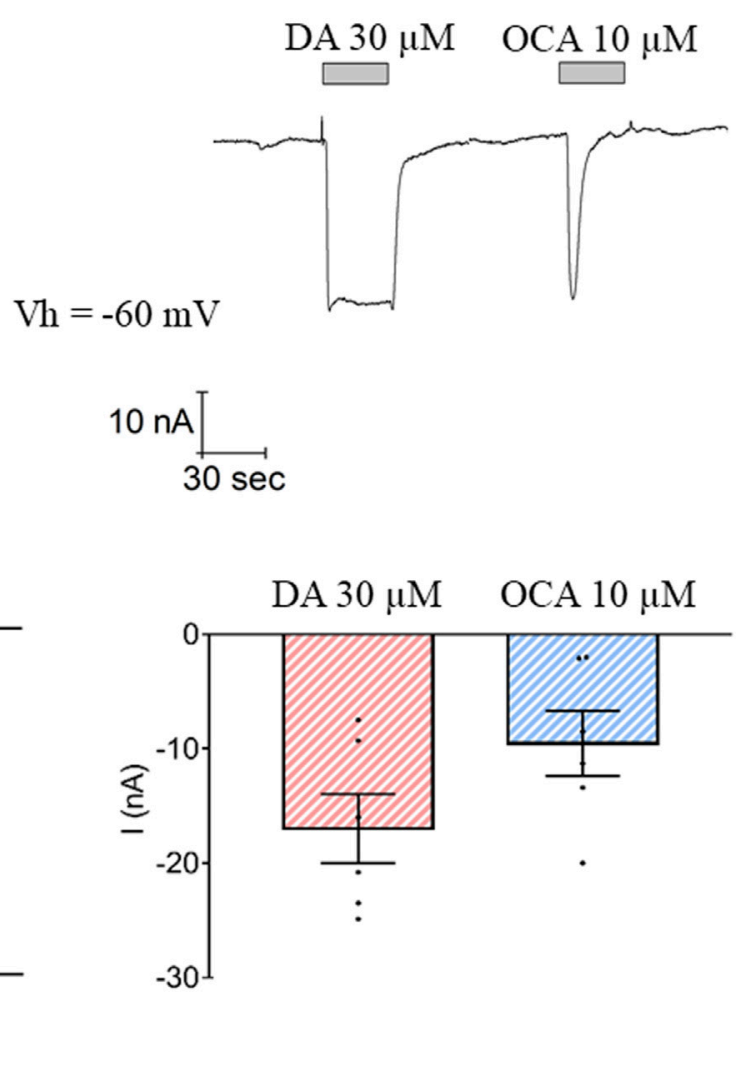

FIGURE 3 | Intracellular calcium does not regulate OCA-induced currents. (A) Schematic representation of the EGTA injection technique. (B) Representative trace of current recorded by TEVC $(\mathrm{Vh}=-60 \mathrm{mV})$ in oocytes expressing mDAT and injected with $13 \mathrm{mM}$ EGTA in intracellular solution 30 min before exposure to $30 \mu \mathrm{M}$ DA or $10 \mu \mathrm{M}$ OCA in ND98 buffer (top) and the mean of maximal transport-associated and OCA-induced currents (bottom) (I nA \pm SE of 6-7 oocytes, 2 batches). (C) Mean of maximal OCA-induced currents in mDAT oocytes with or without injection of EGTA. Two-tailed Student's $t$-test, $p=0.476$.

(Penmatsa et al., 2013; Wang et al., 2015; Cheng and Bahar, 2019). Notably, when DA is present, the top binding poses of OCA do not block the binding of substrate DA (Figure 6B, left panel), such that DA is able to bind in the proximity of OCA. This is consistent with the non-competitive binding of OCA revealed in TEVC experiments (Figure 4).

For the IFo conformer, no high affinity binding is observed to the $\mathrm{EC}$ vestibule. For $\mathrm{OCA}(\mathrm{n})$, the top binding site is in proximity to $\mathrm{D} 421$ within the IC vestibule (Figure 6A, bottom right panel). OCA(-) preferentially binds near the IC entrance, in the proximity to $\mathrm{R} 445$, and no high affinity binding is observed within the vestibule. Notably, docking simulations also revealed binding instances within the transmembrane region, including the site known to be occupied by cholesterol near TM1a (Penmatsa et al., 2013; Cheng and Bahar, 2019), or a site close to residues R443/H444 (Figure 6A, middle left panel) reported previously as a $\mathrm{PIP}_{2}$ binding site (Belovich et al., 2019). However, these sites could potentially be obstructed by lipid molecules, which are not included in docking simulations, and hence will not be further elaborated.
Unexpectedly, in the case of the occluded DAT conformer (Figure 6C), both charged and neutral OCAs $(\mathrm{OCA}(\mathrm{n} /-))$ are predicted to bind to the $\mathrm{S} 1$ site, with almost identical affinity, and the binding pose closely resembles that resolved for ibogainebound hSERT. Of note, the binding pocket in the occluded state may be much larger than anticipated, with possible involvement of TM9 (Figure 6C, right panel).

Taken together, these docking simulations suggest that the binding sites and affinities depend on the net charge carried by OCA and on the conformational state of the transporter. In the OFo state, the binding site for OCA(n) closely neighbors, but does not overlap with, the S1 site resolved for DA (Wang et al., 2015); whereas, in the absence of DA, the binding site for OCA(-) overlaps with the broadly-defined S2 site (Figure 6A). In the IFo state, on the other hand, OCA binds near D421 or R445, depending on its protonation state. Notably, in the occluded state of the transporter, OCA appears to select a binding site similar to that resolved for ibogaine-bound to hSERT (Coleman et al., 2019) regardless of its charge. However, caution is needed when interpreting docking-predicted binding sites, especially in 

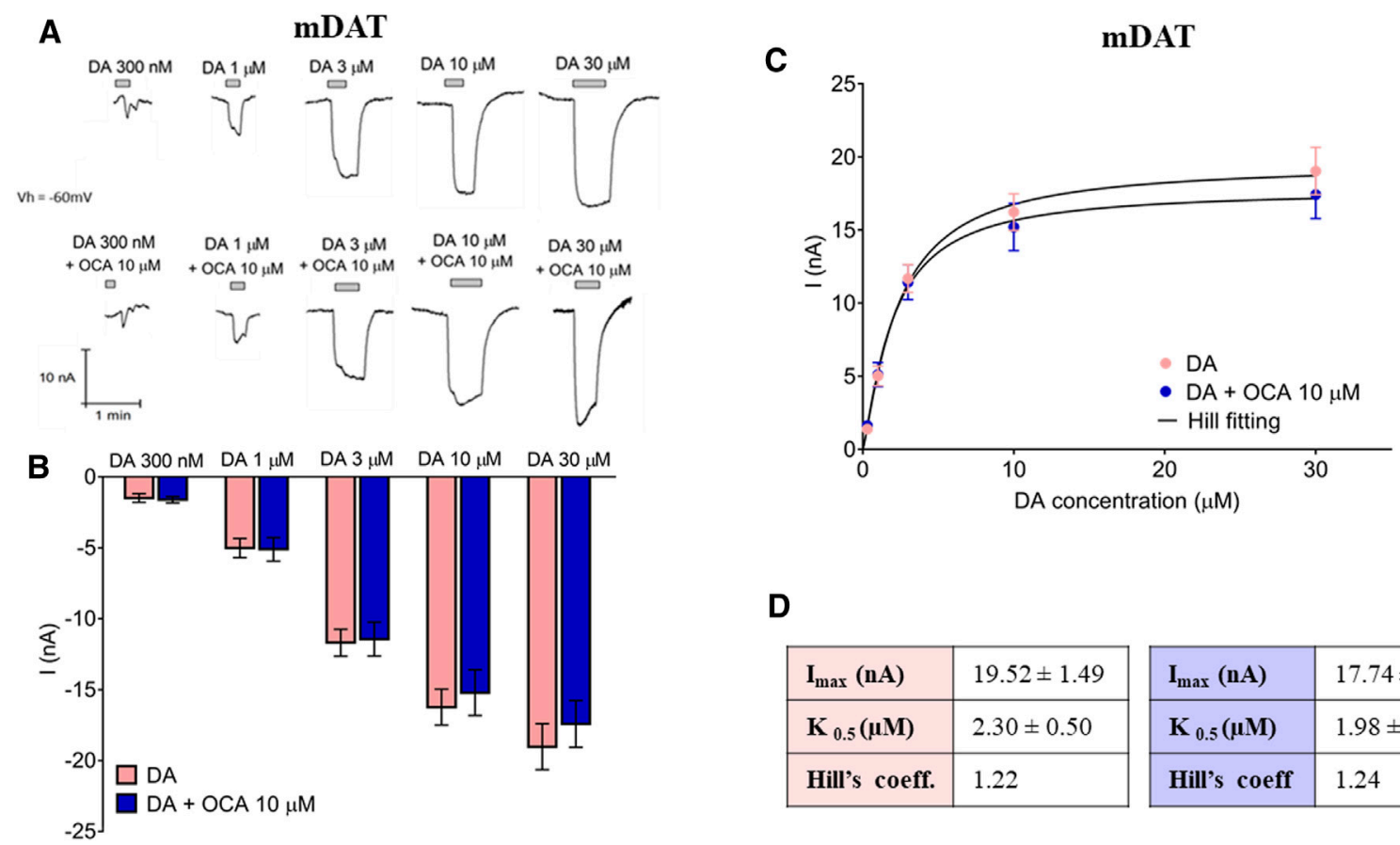

D

\begin{tabular}{|l|l|}
\hline $\mathbf{I}_{\max }(\mathbf{n A})$ & $19.52 \pm 1.49$ \\
\hline $\mathbf{K}_{\mathbf{0 . 5}}(\boldsymbol{\mu M})$ & $2.30 \pm 0.50$ \\
\hline Hill's coeff. & 1.22 \\
\hline
\end{tabular}

\begin{tabular}{|l|l|}
\hline $\mathbf{I}_{\max }(\mathbf{n A})$ & $17.74 \pm 1.57$ \\
\hline $\mathbf{K}_{\mathbf{0 . 5}}(\boldsymbol{\mu M})$ & $1.98 \pm 0.51$ \\
\hline Hill's coeff & 1.24 \\
\hline
\end{tabular}

FIGURE 4 | OCA does not alter the DA transport associated current. (A) Representative traces of the current recorded at increasing concentrations of DA in the absence or presence of $10 \mu \mathrm{M}$ OCA. (B) Mean currents recorded from the traces in (A). Two-way ANOVA, Treatment $\mathrm{F}(1,142)=0.51, p=0.476-\mathrm{DA}$ concentration $F(4,142)=77.76, p<0.0001$ - Interaction $F(4,142)=0.24, p=0.915$. (C) Data from (A) were fitted to a Hill equation. (D) Imax, $K_{0.5}$, and Hill coefficient obtained from fitting the data represented in (B) to the Hill equation.

the occluded state. The S1 site suggested in the occluded DAT conformer may not be accessible to OCA(-) in practice, even though it forms the pocket with the highest affinity, due to the fact that OCA(-) does not bind to the S1 site in the OFo conformer.

\section{DISCUSSION}

The comprehensive role of BAs in brain physiology is still unclear, but evidence of their involvement in various brain functions is continually increasing. BA concentrations in the brain strongly correlate with their plasma concentrations (Mano et al., 2004; Higashi et al., 2017). OCA, the main BA considered in this study, reaches a plasma concentration around $15 \mu \mathrm{M}$ following intraduodenal administration in rats (Roda et al., 2014), comparable to the $10 \mu \mathrm{M}$ concentrations used in bath applications for the TEVC studies reported here. Twenty BAs have been detected in brain lysates and most are present in forms loosely bound to proteins (Higashi et al., 2017; Kiriyama and Nochi, 2019). In contrast, cholesterol, which is highly lipophilic, is only soluble and present in plasma when tightly bound to plasma proteins (Cortes et al., 2014). As a consequence, regulation of DAT via cholesterol occurs upon intercalation into the plasma membrane (Hong and Amara, 2010; Zeppelin et al., 2018). However, the increased charge present on BAs likely precludes this mechanism for regulation of MATs via these molecules. Although our docking simulations revealed, among others, binding instances of OCA to the site known to be occupied by cholesterol (Penmatsa et al., 2013; Cheng and Bahar, 2019), this site is likely obstructed by higher affinity lipid molecules, which are not included in docking simulations, and hence were not further elaborated.

These current findings highlight previously undocumented interactions and impact of BAs on DAT function. Specifically, we show that this interaction promotes a current that is transient in nature and is $\mathrm{Na}^{+}$-dependent. OCA is capable of inhibiting, as observed for DA (Sonders et al., 1997), a DAT-mediated $\mathrm{Li}^{+}$-leak current suggesting that BAs can interact with DAT even in the absence of $\mathrm{Na}^{+}$. Furthermore, the OCA-induced current does not depend upon changes in intracellular calcium levels. In our interpretation, the presence of a DAT-mediated OCA current suggests that OCA could induce an occluded conformation of the transporter. This conformational state could transition to an outward facing conformer upon binding to substrate, as suggested by the competition experiments.

As per the nature of the current itself, the data obtained strongly suggest that the current generated by OCA is a DATmediated, $\mathrm{Na}^{+}$-dependent leak current. In previous in silico studies of DA-free DAT conformers (Cheng et al., 2018; Cheng and Bahar, 2019), the EC- and IC-exposed helices were not as tightly packed as in the occluded DA-bound form. These conformers occasionally gave rise to simultaneous opening of both the EC and IC gates such that formation of an intermittent water channel was detected. Notably, the sodium permeation pathway (Aguilar et al., 2021) coincides with that of water channeling (Cheng et al., 2018; Cheng and Bahar, 2019). This 


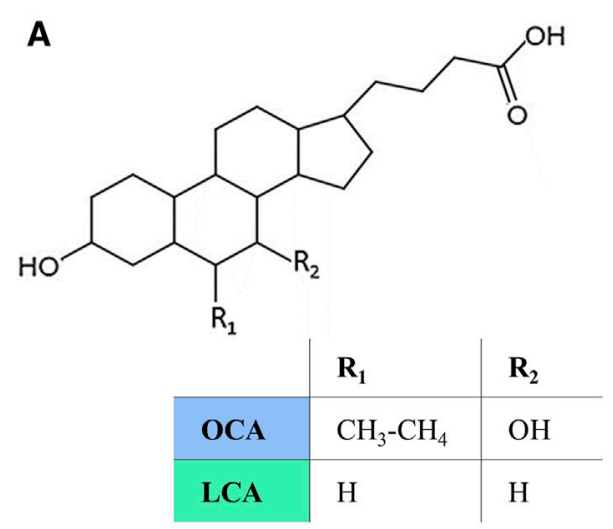

\section{B $\quad$ mDAT}

$\mathrm{Vh}=-60 \mathrm{mV}$

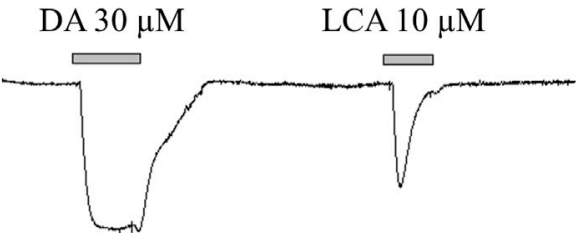

$5 \mathrm{nA} \frac{}{30 \mathrm{sec}}$
C

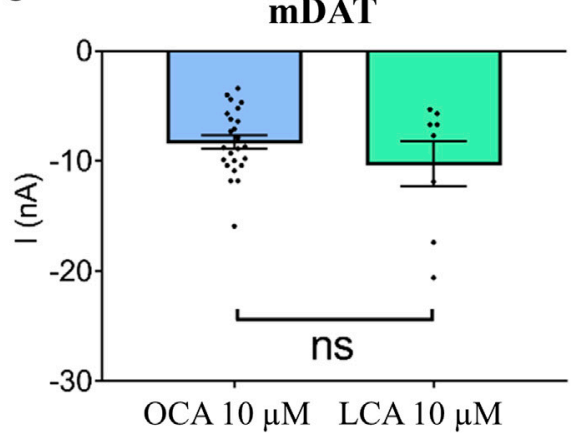

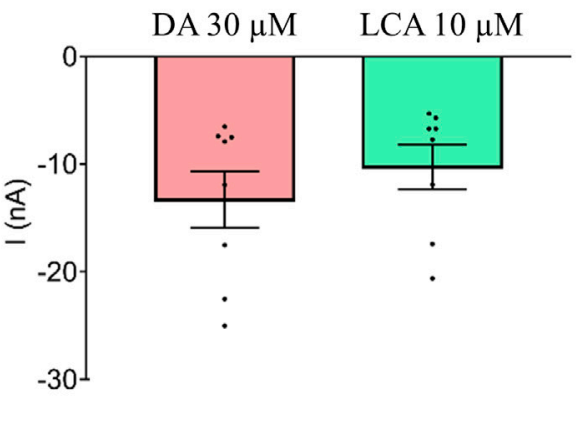

FIGURE 5 | LCA induces a current that parallels the OCA-induced current. (A) Structure of OCA and LCA (B) Representative trace of current recorded with TEVC $(\mathrm{Vh}=-60 \mathrm{mV})$ in oocytes expressing mDAT and perfused with $30 \mu \mathrm{M}$ DA or $10 \mu \mathrm{M}$ LCA in ND98 buffer (top). Mean of maximal dopamine transport-associated and LCA-induced currents (I nA \pm SE of 8 oocytes, 3 batches) (bottom). (C) Mean of maximal currents elicited by OCA or LCA in mDAT-expressing oocytes ( $n=8 ; p=0.22$ by Student's $t$-test).

path, observed in silico, may also be associated with DATmediated ion fluxes or leak currents (Ingram et al., 2002; Erreger et al., 2008). Furthermore, $\mathrm{Na}^{+}$substitution with $\mathrm{TMA}^{+}$strongly reduces the ability of OCA to induce this current. The blocking of the $\mathrm{Li}^{+}$-leak current could be caused by binding of OCA, in the charged state, to the $\mathrm{S} 2$ substrate site, and its deeper insertion and translocation after protonation that may induce intramolecular rearrangements similar to DA. Differences were observed between the effects of OCA and DA on DAT conductance in terms of steady-state currents. This may be due to the fact that the release of DA and simultaneously-bound $\mathrm{Na}^{+}$restores DAT into a transporter mode (Borre et al., 2014), whereas the non-substrate OCA will not facilitate full procession to this state resulting in a current that is transient. Considering that $\mathrm{Li}^{+}$leakage in DAT is dependent on the $\mathrm{Na} 2$ site rather than the $\mathrm{Na} 1$ site (Borre et al., 2014), another possibility is that D421 in hDAT coordinates the binding of the $\mathrm{Na}^{+}$ion to the $\mathrm{Na} 2$ site (Borre et al., 2014; Cheng and Bahar, 2015). Notably, the present docking simulations also indicate that D421 may contribute the binding of OCA(n) (Figure 6A) in the IFo state. The direct binding of OCA to D421 may also potentially block $\mathrm{Li}^{+}$permeation. However, we cannot rule out the possibility that inhibition of the $\mathrm{Li}^{+}$current by OCA may reflect a shift of conformational equilibrium between different functional states along the transport cycle, as proposed for DA (Borre et al., 2014).

The docking simulations also provide important insights into the potential functional relevance of the OCA-DAT interaction. Surprisingly, these results suggest that OCA binds a similar site to that resolved for ibogaine-bound hSERT (Coleman et al., 2019) in the occluded state (Figure 6C). Ibogaine is a non-competitive inhibitor for both DAT and SERT and has been proposed to stabilize the transporters in the IF conformation (Jacobs et al., 2007; Bulling et al., 2012). It is important to consider that compounds that bind to the S1 or S2 site (or at both sites) would induce an inhibition of substrate transport. However, we observed that OCA binds in a non-competitive way with respect to DA (Figure 4). One possibility is that the predominant form of OCA present in experiments carried a negative-charge and therefore did not bind the S1 site as modeled for OCA $(-)$ in the OFo state (Figure 6, panel A). The negatively-charged OCA, in fact, binds only superficially near the entrance to the EC vestibule of DAT and could only exert a limited effect on DA transport. Further studies on the residues involved in the binding will aid in clarification of this point.

Of note, our model points to a separate site, of equal potential functional impact, for OCA on DAT; residues R445 and D436 (Figure 6A) which are involved in the formation of salt bridges in 

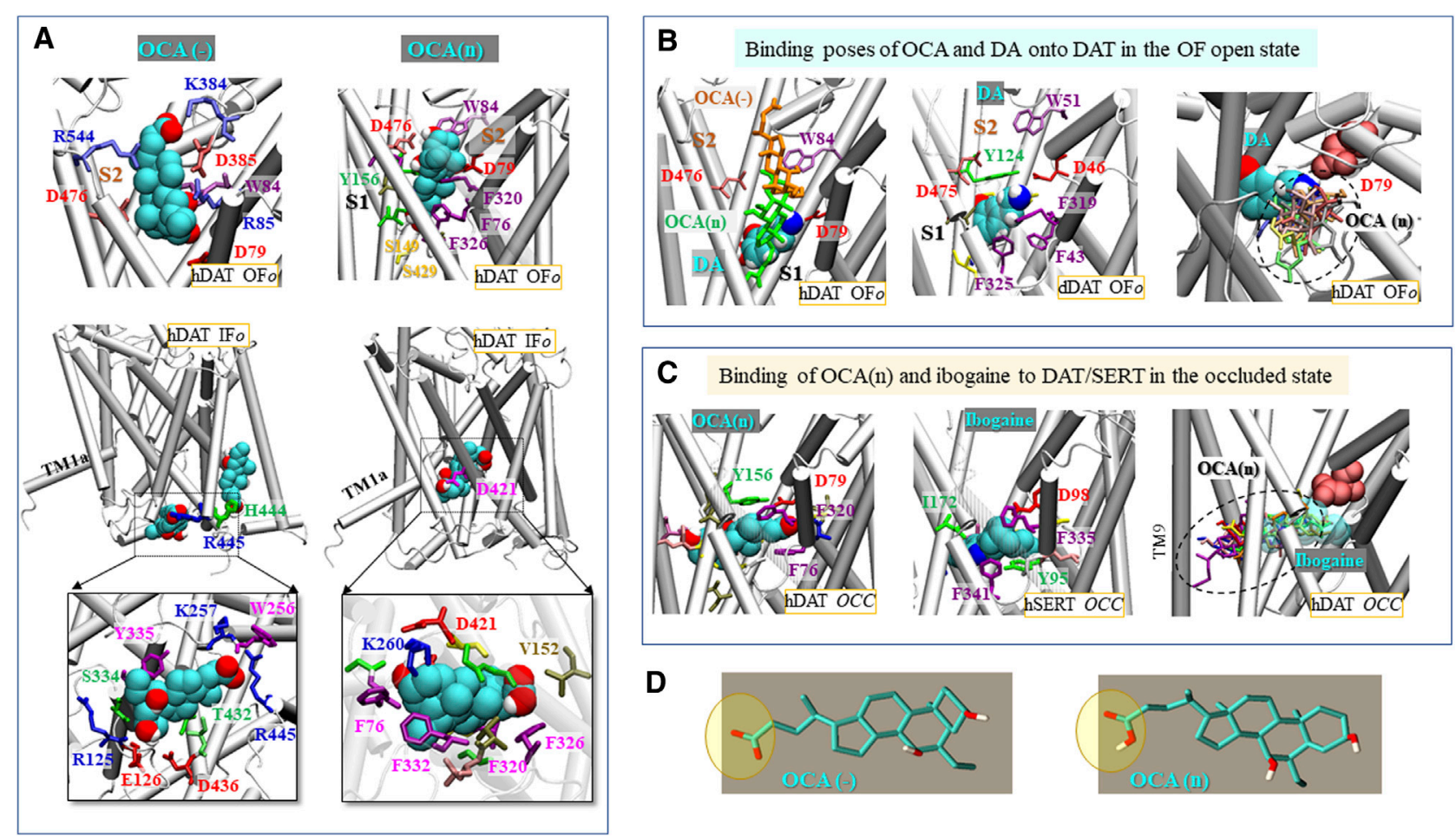

D
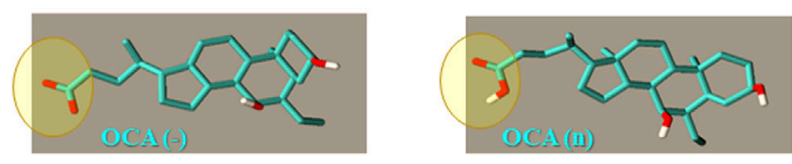

FIGURE 6 | OCA binding to DAT depends on its protonation state and on DAT conformation. (A) Binding of negatively charged (left) and neutral (right) OCA (cyan) to hDAT in the OFo (top two panels) and IFO (middle and bottom panels) states. The binding energies to OFo hDAT are $-6.1 \mathrm{kcal} / \mathrm{mol}(\mathrm{OCA}(-))$ and $-9.9 \mathrm{kcal} / \mathrm{mol}$ $(\mathrm{OCA}(\mathrm{n}))$; and those to the IFo $\mathrm{hDAT}$ are $-7.5 \mathrm{kcal} / \mathrm{mol}(\mathrm{OCA}(-))$ and $-8.5 \mathrm{kcal} / \mathrm{mol}(\mathrm{OCA}(\mathrm{n}))$. Interacting residues making atom-atom contacts closer than $4 \AA$ with $\mathrm{OCA}$ are shown in panels (A-C). (B) Comparison with the known DA-binding pose. Alignment of the top binding poses of OCA(n) (green sticks), OCA(-) (orange sticks) (left panel) and the resolved DA (van der Waals (vdW) format) bound dDAT (PDB: 4XP1) (middle panel), and detailed view of DA-binding site and comparison of the non-overlapping spaces occupied by OCA (multiple binding poses) and DA (right panel). (C) Top 1 binding pose of OCA(n) (vdW format; -10.2 kcal/mol) to hDAT occluded (OCC) conformer (left pane), the resolved ibogaine-bound to hSERT in the OCC state (PDB:6DZV) (middle panel) and alignment, onto hDAT, of top 10 binding poses of OCA(n) (sticks in different colors; average $=-9.1 \pm 1.0 \mathrm{kcal} / \mathrm{mol}$ ) and ibogaine bound to SERT (transparent vDW) (right panel). OCA, DA, and ibogaine are shown in vdW format in (A-C), with cyan, red, blue and white spheres representing the carbon, oxygen, nitrogen and hydrogen atoms, respectively. (D) Molecular structures of OCA in two protonation states.

the IF conformer. In the DAT transport cycle, the binding of DA to S2 and S1 promotes the closure of the EC gate upon stabilization in the site S1. It then cooperatively restores the compact association of the EC vestibule while translocating to the IC vestibule (Cheng and Bahar, 2015). The IC vestibule opens to the cytoplasm in the IF conformation only after compaction/closure of the EC-exposed region (Cheng and Bahar, 2015). The binding of OCA may stabilize the IFo state by preventing the re-formation of the IF gating salt bridges, R60-D436 and R445-E428, that are required for DA transport (Cheng and Bahar, 2019). Recently, molecular modeling found that the infantile Parkinsonism-Dystonia associated substitution, $\mathrm{R} 445 \mathrm{C}$ in hDAT, disrupted a phylogenetically conserved intracellular network of interactions and promoted a channel-like intermediate of hDAT. These rearrangements lead to the permeation of $\mathrm{Na}^{+}$from both the EC and IC solutions (Aguilar et al., 2021). Interestingly, docking simulations suggested that OCA could also bind near H444/R445 to stabilize the IF state or act as an anion-lipid (Figure 6A for $\mathrm{OCA}(-)$ in the IFo state). Further experiments will be performed to confirm the importance of these predicted docking sites for the interaction of OCA and DAT.
Lastly, the findings reported in Figure $\mathbf{5}$ confirm that binding to DAT is not unique to OCA, but takes place also upon application of LCA, a natural bile acid. This observation is important as this class of molecules have not been previously reported to interact with MATs. Thus, BAs in general hold potential as novel pharmacological tools that could potentially impact important MAT functions, such as the channel-like activity of DAT.

\section{DATA AVAILABILITY STATEMENT}

The datasets presented in this study can be found in online repositories. The names of the repository/repositories and accession number(s) can be found in the article.

\section{ETHICS STATEMENT}

The animal study was reviewed and approved by the Committee of the "Organismo Preposto al Benessere degli Animali" of the University of Insubria (OPBA-permit \#02_15) and nationally by Ministero della Salute (permit nr. 1011/2015). 


\section{AUTHOR CONTRIBUTIONS}

TR, DZ, MC, and BS performed experiments, analyzed data, prepared figures and contributed to writing the manuscript. AC contributed to data analysis, writing and editing the manuscript. AG, IB, and EB designed and supervised the studies.

\section{REFERENCES}

Adkins, E. M., Samuvel, D. J., Fog, J. U., Eriksen, J., Jayanthi, L. D., Vaegter, C. B., et al. (2007). Membrane Mobility and Microdomain Association of the Dopamine Transporter Studied with Fluorescence Correlation Spectroscopy and Fluorescence Recovery after Photobleaching. Biochemistry 46, 10484-10497. doi:10.1021/bi700429z

Aggarwal, S., Cheng, M. H., Salvino, J. M., Bahar, I., and Mortensen, O. V. (2021). Functional Characterization of the Dopaminergic Psychostimulant Sydnocarb as an Allosteric Modulator of the Human Dopamine Transporter. Biomedicines 9, 634. doi:10.3390/biomedicines9060634

Aguilar, J. I., Cheng, M. H., Font, J., Schwartz, A. C., Ledwitch, K., Duran, A., et al. (2021). Psychomotor Impairments and Therapeutic Implications Revealed by a Mutation Associated with Infantile Parkinsonism-Dystonia. eLife 10, e68039. doi:10.7554/eLife.68039

Andrini, O., Ghezzi, C., Murer, H., and Forster, I. C. (2008). The Leak Mode of Type II Na+-Pi Cotransporters. Channels 2, 346-357. doi:10.4161/ chan.2.5.6900

Belovich, A. N., Aguilar, J. I., Mabry, S. J., Cheng, M. H., Zanella, D., Hamilton, P. J., et al. (2019). A Network of Phosphatidylinositol (4,5)-Bisphosphate (PIP2) Binding Sites on the Dopamine Transporter Regulates Amphetamine Behavior in Drosophila Melanogaster. Mol. Psychiatry 26, 4417-4430. doi:10.1038/ s41380-019-0620-0

Bensalem, A., Murtaza, B., Hichami, A., Khan, A. S., Oulamara, H., Merlen, G., et al. (2020). Bile Acid Receptor TGR5 Is Critically Involved in Preference for Dietary Lipids and Obesity. J. Nutr. Biochem. 76, 108298. doi:10.1016/ j.jnutbio.2019.108298

Bhargava, P., Smith, M. D., Mische, L., Harrington, E., Fitzgerald, K. C., Martin, K., et al. (2020). Bile Acid Metabolism Is Altered in Multiple Sclerosis and Supplementation Ameliorates Neuroinflammation. J. Clin. Invest. 130, 3467-3482. doi:10.1172/jci129401

Borre, L., Andreassen, T. F., Shi, L., Weinstein, H., and Gether, U. (2014). The Second Sodium Site in the Dopamine Transporter Controls Cation Permeation and Is Regulated by Chloride. J. Biol. Chem. 289, 25764-25773. doi:10.1074/ jbc.m114.574269

Bossi, E., Centinaio, E., Castagna, M., Giovannardi, S., Vincenti, S., Sacchi, V. F., et al. (1999). Ion Binding and Permeation through the Lepidopteran Amino Acid Transporter KAAT1 Expressed in Xenopus Oocytes. J. Physiol. 515 (Pt 3), 729-742. doi:10.1111/j.1469-7793.1999.729ab.x

Bossi, E., Fabbrini, M. S., and Ceriotti, A. (2007). Exogenous Protein Expression in Xenopus Oocytes. Methods Mol. Biol. 375, 107-131. doi:10.1007/978-1-59745388-2_6

Bulling, S., Schicker, K., Zhang, Y.-W., Steinkellner, T., Stockner, T., Gruber, C. W., et al. (2012). The Mechanistic Basis for Noncompetitive Ibogaine Inhibition of Serotonin and Dopamine Transporters. J. Biol. Chem. 287, 18524-18534. doi:10.1074/jbc.m112.343681

Butchbach, M. E. R., Tian, G., Guo, H., and Lin, C.-1. G. (2004). Association of Excitatory Amino Acid Transporters, Especially EAAT2, with Cholesterol-Rich Lipid Raft Microdomains. J. Biol. Chem. 279, 34388-34396. doi:10.1074/ jbc.m403938200

Chang, J. C., and Rosenthal, S. J. (2012). Visualization of Lipid Raft Membrane Compartmentalization in Living RN46A Neuronal Cells Using Single Quantum Dot Tracking. ACS Chem. Neurosci. 3, 737-743. doi:10.1021/cn3000845

Chaudhari, S. N., Harris, D. A., Aliakbarian, H., Luo, J. N., Henke, M. T., Subramaniam, R., et al. (2021). Bariatric Surgery Reveals a Gut-Restricted TGR5 Agonist with Anti-diabetic Effects. Nat. Chem. Biol. 17, 20-29. doi:10.1038/s41589-020-0604-z

\section{FUNDING}

This work was supported by NIH awards DA043960 and DA035263 (to AG), P41GM103712 and R01 GM239197 (to IB), R56MH121453 (to IB and MC), and an NSF TECBio REU program award (to BS).

Cheng, M. H., and Bahar, I. (2015). Molecular Mechanism of Dopamine Transport by Human Dopamine Transporter. Structure 23, 2171-2181. doi:10.1016/ j.str.2015.09.001

Cheng, M. H., and Bahar, I. (2019). Monoamine Transporters: Structure, Intrinsic Dynamics and Allosteric Regulation. Nat. Struct. Mol. Biol. 26, 545-556. doi:10.1038/s41594-019-0253-7

Cheng, M. H., Block, E., Hu, F., Cobanoglu, M. C., Sorkin, A., and Bahar, I. (2015). Insights into the Modulation of Dopamine Transporter Function by Amphetamine, Orphenadrine, and Cocaine Binding. Front. Neurol. 6, 134 doi:10.3389/fneur.2015.00134

Cheng, M. H., Kaya, C., and Bahar, I. (2018). Quantitative Assessment of the Energetics of Dopamine Translocation by Human Dopamine Transporter. J. Phys. Chem. B 122, 5336-5346. doi:10.1021/acs.jpcb.7b10340

Coleman, J. A., Green, E. M., and Gouaux, E. (2016). X-Ray Structures and Mechanism of the Human Serotonin Transporter. Nature 532, 334-339. doi:10.1038/nature17629

Coleman, J. A., Yang, D., Zhao, Z., Wen, P.-C., Yoshioka, C., Tajkhorshid, E., et al. (2019). Serotonin Transporter-Ibogaine Complexes Illuminate Mechanisms of Inhibition and Transport. Nature 569, 141-145. doi:10.1038/s41586-0191135-1

Cortes, V., Busso, D., Maiz, A., Arteaga, A., Nervi, F., and Rigotti, A. (2014). Physiological and Pathological Implications of Cholesterol. Front. Biosci. 19, 416-428. doi: $10.2741 / 4216$

Cremona, M. L., Matthies, H. J. G., Pau, K., Bowton, E., Speed, N., Lute, B. J., et al. (2011). Flotillin-1 Is Essential for PKC-Triggered Endocytosis and Membrane Microdomain Localization of DAT. Nat. Neurosci. 14, 469-477. doi:10.1038/ nn. 2781

Cruz-Ramón, V., Chinchilla-López, P., Ramírez-Pérez, O., and Méndez-Sánchez, N. (2017). Bile Acids in Nonalcoholic Fatty Liver Disease: New Concepts and Therapeutic Advances. Ann. Hepatol. 16 (Suppl. 1), S58-S67. doi:10.5604/ 01.3001 .0010 .5498

Erreger, K., Grewer, C., Javitch, J. A., and Galli, A. (2008). Currents in Response to Rapid Concentration Jumps of Amphetamine Uncover Novel Aspects of Human Dopamine Transporter Function. J. Neurosci. 28, 976-989. doi:10.1523/jneurosci.2796-07.2008

Fiorucci, S., Distrutti, E., Carino, A., Zampella, A., and Biagioli, M. (2021). Bile Acids and Their Receptors in Metabolic Disorders. Prog. Lipid Res. 82, 101094 doi:10.1016/j.plipres.2021.101094

Flynn, C. R., Albaugh, V. L., and Abumrad, N. N. (2019). Metabolic Effects of Bile Acids: Potential Role in Bariatric Surgery. Cell Mol. Gastroenterol. Hepatol. 8, 235-246. doi:10.1016/j.jcmgh.2019.04.014

Foster, J. D., Adkins, S. D., Lever, J. R., and Vaughan, R. A. (2008). Phorbol Ester Induced Trafficking-independent Regulation and Enhanced Phosphorylation of the Dopamine Transporter Associated with Membrane Rafts and Cholesterol. J. Neurochem. 105, 1683-1699. doi:10.1111/j.14714159.2008.05262.x

Giros, B., El Mestikawy, S., Godinot, N., Zheng, K., Han, H., Yang-Feng, T., et al. (1992). Cloning, Pharmacological Characterization, and Chromosome Assignment of the Human Dopamine Transporter. Mol. Pharmacol. 42, 383-390.

Hao, H., Cao, L., Jiang, C., Che, Y., Zhang, S., Takahashi, S., et al. (2017). Farnesoid $\mathrm{X}$ Receptor Regulation of the NLRP3 Inflammasome Underlies CholestasisAssociated Sepsis. Cel Metab. 25, 856-867. doi:10.1016/j.cmet.2017.03.007

Higashi, T., Watanabe, S., Tomaru, K., Yamazaki, W., Yoshizawa, K., Ogawa, S., et al. (2017). Unconjugated Bile Acids in Rat Brain: Analytical Method Based on LC/ESI-MS/MS with Chemical Derivatization and Estimation of Their Origin by Comparison to Serum Levels. Steroids 125, 107-113. doi:10.1016/ j.steroids.2017.07.001 
Hong, W. C., and Amara, S. G. (2010). Membrane Cholesterol Modulates the Outward Facing Conformation of the Dopamine Transporter and Alters Cocaine Binding. J. Biol. Chem. 285, 32616-32626. doi:10.1074/ jbc.m110.150565

Huang, F., Wang, T., Lan, Y., Yang, L., Pan, W., Zhu, Y., et al. (2015). Deletion of Mouse FXR Gene Disturbs Multiple Neurotransmitter Systems and Alters Neurobehavior. Front. Behav. Neurosci. 9, 70. doi:10.3389/ fnbeh.2015.00070

Huang, F., Pariante, C. M., and Borsini, A. (2021). From Dried bear Bile to Molecular Investigation: A Systematic Review of the Effect of Bile Acids on Cell Apoptosis, Oxidative Stress and Inflammation in the Brain, across Pre-Clinical Models of Neurological, Neurodegenerative and Neuropsychiatric Disorders. Brain Behav. Immun. 99, 132-146. doi:10.1016/j.bbi.2021.09.021

Ingram, S. L., Prasad, B. M., and Amara, S. G. (2002). Dopamine TransporterMediated Conductances Increase Excitability of Midbrain Dopamine Neurons. Nat. Neurosci. 5, 971-978. doi:10.1038/nn920

Irwin, J. J., and Shoichet, B. K. (2005). ZINC - A Free Database of Commercially Available Compounds for Virtual Screening. J. Chem. Inf. Model. 45, 177-182. doi:10.1021/ci049714+

Jacobs, M. T., Zhang, Y.-W., Campbell, S. D., and Rudnick, G. (2007). Ibogaine, a Noncompetitive Inhibitor of Serotonin Transport, Acts by Stabilizing the Cytoplasm-Facing State of the Transporter. J. Biol. Chem. 282, 29441-29447. doi:10.1074/jbc.m704456200

James-Zorn, C., Ponferrada, V., Fisher, M. E., Burns, K., Fortriede, J., Segerdell, E., et al. (2018). Navigating Xenbase: An Integrated Xenopus Genomics and Gene Expression Database. Methods Mol. Biol. 1757, 251-305. doi:10.1007/978-14939-7737-6_10

Jin, P., Deng, S., Tian, M., Lenahan, C., Wei, P., Wang, Y., et al. (2021). INT-777 Prevents Cognitive Impairment by Activating Takeda G Protein-Coupled Receptor 5 (TGR5) and Attenuating Neuroinflammation via cAMP/PKA/ CREB Signaling axis in a Rat Model of Sepsis. Exp. Neurol. 335, 113504. doi:10.1016/j.expneurol.2020.113504

Jones, K. T., Zhen, J., and Reith, M. E. A. (2012). Importance of Cholesterol in Dopamine Transporter Function. J. Neurochem. 123, 700-715. doi:10.1111/ jnc. 12007

Kawamata, Y., Fujii, R., Hosoya, M., Harada, M., Yoshida, H., Miwa, M., et al. (2003). A G Protein-Coupled Receptor Responsive to Bile Acids. J. Biol. Chem. 278, 9435-9440. doi:10.1074/jbc.m209706200

Keitel, V., Görg, B., Bidmon, H. J., Zemtsova, I., Spomer, L., Zilles, K., et al. (2010). The Bile Acid Receptor TGR5 (Gpbar-1) Acts as a Neurosteroid Receptor in Brain. Glia 58, 1794-1805. doi:10.1002/glia.21049

Kiriyama, Y., and Nochi, H. (2019). The Biosynthesis, Signaling, and Neurological Functions of Bile Acids. Biomolecules 9, 232. doi:10.3390/biom9060232

Lester, H. A., Mager, S., Quick, M. W., and Corey, J. L. (1994). Permeation Properties of Neurotransmitter Transporters. Annu. Rev. Pharmacol. Toxicol. 34, 219-249. doi:10.1146/annurev.pa.34.040194.001251

Li, T., and Chiang, J. Y. L. (2020). Bile Acid-Based Therapies for Non-Alcoholic Steatohepatitis and Alcoholic Liver Disease. Hepatobiliary Surg. Nutr. 9, 152-169. doi:10.21037/hbsn.2019.09.03

Lieu, T., Jayaweera, G., Zhao, P., Poole, D. P., Jensen, D., Grace, M., et al. (2014). The Bile Acid Receptor TGR5 Activates the TRPA1 Channel to Induce Itch in Mice. Gastroenterology 147, 1417-1428. doi:10.1053/ j.gastro.2014.08.042

Magnani, F., Tate, C. G., Wynne, S., Williams, C., and Haase, J. (2004). Partitioning of the Serotonin Transporter into Lipid Microdomains Modulates Transport of Serotonin. J. Biol. Chem. 279, 38770-38778. doi:10.1074/jbc.m400831200

Mano, N., Goto, T., Uchida, M., Nishimura, K., Ando, M., Kobayashi, N., et al. (2004). Presence of Protein-Bound Unconjugated Bile Acids in the Cytoplasmic Fraction of Rat Brain. J. Lipid Res. 45, 295-300. doi:10.1194/ jlr.m300369-jlr200

Maruyama, T., Miyamoto, Y., Nakamura, T., Tamai, Y., Okada, H., Sugiyama, E., et al. (2002). Identification of Membrane-Type Receptor for Bile Acids (M-BAR). Biochem. Biophysical Res. Commun. 298, 714-719. doi:10.1016/ s0006-291x(02)02550-0

McMillin, M., and DeMorrow, S. (2016). Effects of Bile Acids on Neurological Function and Disease. FASEB j. 30, 3658-3668. doi:10.1096/fi.201600275r
Mertens, K. L., Kalsbeek, A., Soeters, M. R., and Eggink, H. M. (2017). Bile Acid Signaling Pathways from the Enterohepatic Circulation to the Central Nervous System. Front. Neurosci. 11, 617. doi:10.3389/fnins.2017.00617

Morris, G. M., Huey, R., Lindstrom, W., Sanner, M. F., Belew, R. K., Goodsell, D. S., et al. (2009). AutoDock4 and AutoDockTools4: Automated Docking with Selective Receptor Flexibility. J. Comput. Chem. 30, 2785-2791. doi:10.1002/ jcc. 21256

Penmatsa, A., Wang, K. H., and Gouaux, E. (2013). X-Ray Structure of Dopamine Transporter Elucidates Antidepressant Mechanism. Nature 503, 85-90. doi:10.1038/nature12533

Perino, A., Velázquez-Villegas, L. A., Bresciani, N., Sun, Y., Huang, Q., Fénelon, V. S., et al. (2021). Central Anorexigenic Actions of Bile Acids Are Mediated by TGR5. Nat. Metab. 3, 595-603. doi:10.1038/s42255-021-00398-4

Raufman, J.-P., Chen, Y., Cheng, K., Compadre, C., Compadre, L., and Zimniak, P. (2002). Selective Interaction of Bile Acids with Muscarinic Receptors: A Case of Molecular Mimicry. Eur. J. Pharmacol. 457, 77-84. doi:10.1016/s0014-2999(02) 02690-0

Reddy, I. A., Smith, N. K., Erreger, K., Ghose, D., Saunders, C., Foster, D. J., et al. (2018). Bile Diversion, a Bariatric Surgery, and Bile Acid Signaling Reduce Central Cocaine Reward. Plos Biol. 16, e2006682. doi:10.1371/ journal.pbio.2006682

Roda, A., Pellicciari, R., Gioiello, A., Neri, F., Camborata, C., Passeri, D., et al. (2014). Semisynthetic Bile Acid FXR and TGR5 Agonists: Physicochemical Properties, Pharmacokinetics, and Metabolism in the Rat. J. Pharmacol. Exp. Ther. 350, 56-68. doi:10.1124/jpet.114.214650

Russell, D. W. (2003). The Enzymes, Regulation, and Genetics of Bile Acid Synthesis. Annu. Rev. Biochem. 72, 137-174. doi:10.1146/ annurev.biochem.72.121801.161712

Scholtz, S., Miras, A. D., Chhina, N., Prechtl, C. G., Sleeth, M. L., Daud, N. M., et al. (2014). Obese Patients after Gastric Bypass Surgery Have Lower Brain-Hedonic Responses to Food Than after Gastric Banding. Gut 63, 891-902. doi:10.1136/ gutjnl-2013-305008

Schubring, S. R., Fleischer, W., Lin, J. S., Haas, H. L., and Sergeeva, O. A. (2012) The Bile Steroid Chenodeoxycholate Is a Potent Antagonist at NMDA and GABAA Receptors. Neurosci. Lett. 506, 322-326. doi:10.1016/ j.neulet.2011.11.036

Session, A. M., Uno, Y., Kwon, T., Chapman, J. A., Toyoda, A., Takahashi, S., et al. (2016). Genome Evolution in the Allotetraploid Frog Xenopus L. Nature 538, 336-343. doi:10.1038/nature19840

Sonders, M. S., Zhu, S.-J., Zahniser, N. R., Kavanaugh, M. P., and Amara, S. G. (1997). Multiple Ionic Conductances of the Human Dopamine Transporter: The Actions of Dopamine and Psychostimulants. J. Neurosci. 17, 960-974. doi:10.1523/jneurosci.17-03-00960.1997

Trott, O., and Olson, A. J. (2010). AutoDock Vina: Improving the Speed and Accuracy of Docking with a New Scoring Function, Efficient Optimization, and Multithreading. J. Comput. Chem. 31, 455-461. doi:10.1002/jcc.21334

Wang, K. H., Penmatsa, A., and Gouaux, E. (2015). Neurotransmitter and Psychostimulant Recognition by the Dopamine Transporter. Nature 521, 322-327. doi:10.1038/nature14431

Waterhouse, A., Bertoni, M., Bienert, S., Studer, G., Tauriello, G., Gumienny, R., et al. (2018). SWISS-MODEL: Homology Modelling of Protein Structures and Complexes. Nucleic Acids Res. 46, W296-w303. doi:10.1093/nar/gky427

Wiemuth, D., Assmann, M., and Gründer, S. (2014). The Bile Acid-Sensitive Ion Channel (BASIC), the Ignored Cousin of ASICs and ENaC. Channels 8, 29-34. doi:10.4161/chan.27493

Wishart, D. S., Knox, C., Guo, A. C., Shrivastava, S., Hassanali, M., Stothard, P., et al. (2006). DrugBank: A Comprehensive Resource for In Silico Drug Discovery and Exploration. Nucleic Acids Res. 34, D668-D672. doi:10.1093/ nar/gkj067

Wu, H., Yu, N., Wang, X., Yang, Y., and Liang, H. (2020). Tauroursodeoxycholic Acid Attenuates Neuronal Apoptosis via the TGR5/SIRT3 Pathway after Subarachnoid Hemorrhage in Rats. Biol. Res. 53, 56. doi:10.1186/s40659020-00323-1

Yanai, I., Peshkin, L., Jorgensen, P., and Kirschner, M. W. (2011). Mapping Gene Expression in Two Xenopus Species: Evolutionary Constraints and Developmental Flexibility. Develop. Cel 20, 483-496. doi:10.1016/ j.devcel.2011.03.015 
Zangerolamo, L., Vettorazzi, J. F., Rosa, L. R. O., Carneiro, E. M., and Barbosa, H. C. L. (2021). The Bile Acid TUDCA and Neurodegenerative Disorders: An Overview. Life Sci. 272, 119252. doi:10.1016/j.lfs.2021.119252

Zeppelin, T., Ladefoged, L. K., Sinning, S., Periole, X., and Schiøtt, B. (2018). A Direct Interaction of Cholesterol with the Dopamine Transporter Prevents its Out-ToInward Transition. Plos Comput. Biol. 14, e1005907. doi:10.1371/journal.pcbi.1005907

Conflict of Interest: The authors declare that the research was conducted in the absence of any commercial or financial relationships that could be construed as a potential conflict of interest.

The reviewer $(\mathrm{PH})$ declared a past co-authorship with the authors (DZ, MC) to the handling Editor.
Publisher's Note: All claims expressed in this article are solely those of the authors and do not necessarily represent those of their affiliated organizations, or those of the publisher, the editors and the reviewers. Any product that may be evaluated in this article, or claim that may be made by its manufacturer, is not guaranteed or endorsed by the publisher.

Copyright () 2021 Romanazzi, Zanella, Cheng, Smith, Carter, Galli, Bahar and Bossi. This is an open-access article distributed under the terms of the Creative Commons Attribution License (CC BY). The use, distribution or reproduction in other forums is permitted, provided the original author(s) and the copyright owner(s) are credited and that the original publication in this journal is cited, in accordance with accepted academic practice. No use, distribution or reproduction is permitted which does not comply with these terms. 